\title{
Open problems in some competition models
}

\author{
Saber Elaydi \& Rafael Luís
}

To cite this article: Saber Elaydi \& Rafael Luís (2011) Open problems in some competition models, Journal of Difference Equations and Applications, 17:12, 1873-1877, DOI: 10.1080/10236198.2011.559468

To link to this article: https://doi.org/10.1080/10236198.2011.559468

Published online: 22 Mar 2011.

[

Џll Article views: 854

Q View related articles $\square$

7 Citing articles: 5 View citing articles $\square$ 


\title{
OPEN PROBLEMS AND CONJECTURES
}

\section{Open problems in some competition models}

\author{
Saber Elaydi ${ }^{\mathrm{a} 1}$ and Rafael Luís ${ }^{\mathrm{b} *}$ \\ ${ }^{a}$ Department of Mathematics, Trinity University, San Antonio, TX, USA; ${ }^{b}$ Center for Mathematical \\ Analysis, Geometry, and Dynamical Systems, Instituto Superior Tecnico, Technical University of \\ Lisbon, Lisbon, Portugal
}

(Received 22 January 2011; final version received 27 January 2011)

\begin{abstract}
We present open problems and conjectures for some two-dimensional competition
\end{abstract} models, namely the logistic competition model and a Ricker-type competition model.

Keywords: Ricker competition model; logistic competition model; stability

\section{Ricker competition model}

In [2], the authors considered the Ricker competition model given by

$$
\left\{\begin{array}{l}
x_{n+1}=x_{n} \mathrm{e}^{K-x_{n}-a y_{n}} \\
y_{n+1}=y_{n} \mathrm{e}^{L-y_{n}-b x_{n}},
\end{array}\right.
$$

where $(x, y) \in \mathbb{R}_{+}^{2}$. The parameters $K, L>0$ are the carrying capacities of species $x$ and $y$, respectively. Moreover, the competition parameters $a$ and $b$ are in the interval $(0,1)$. System (1) is based on the popular 1D Ricker map $R(x)=x \mathrm{e}^{p-x}, p>0$. The following result is the main stability theorem in [2].

THEOREM 1.1. [2] The coexistence fixed point

$$
\left(x^{*}, y^{*}\right)=\left(\frac{a L-K}{a b-1}, \frac{b K-L}{a b-1}\right)
$$

of the Ricker equation (1) is asymptotically stable if

$$
4(a b-1)+2(1-a) L+2(1-b) K \leq(a L-K)(b K-L)<(1-a) L+(1-b) K .
$$

Equivalently, the coexistence fixed point is asymptotically stable if $(K, L) \in \operatorname{Int}\left(S_{1}\right)$, with $a, b \in(0,1)$, and $\operatorname{Int}\left(S_{1}\right)$ denotes the interior of the region $S_{1}$.

The region $S_{1}$, in the parameter space $K-L$, representing (2), is depicted in Figure 1.

Now, we have the following conjecture.

CONJECTURE 1.2. Under condition (2), the coexistence fixed point $\left(x^{*}, y^{*}\right)$ is globally asymptotically stable in the positive first quadrant, for all $(K, L) \in \operatorname{Int}\left(S_{1}\right)$.

*Corresponding author. Email: rafael.luis.madeira@gmail.com 


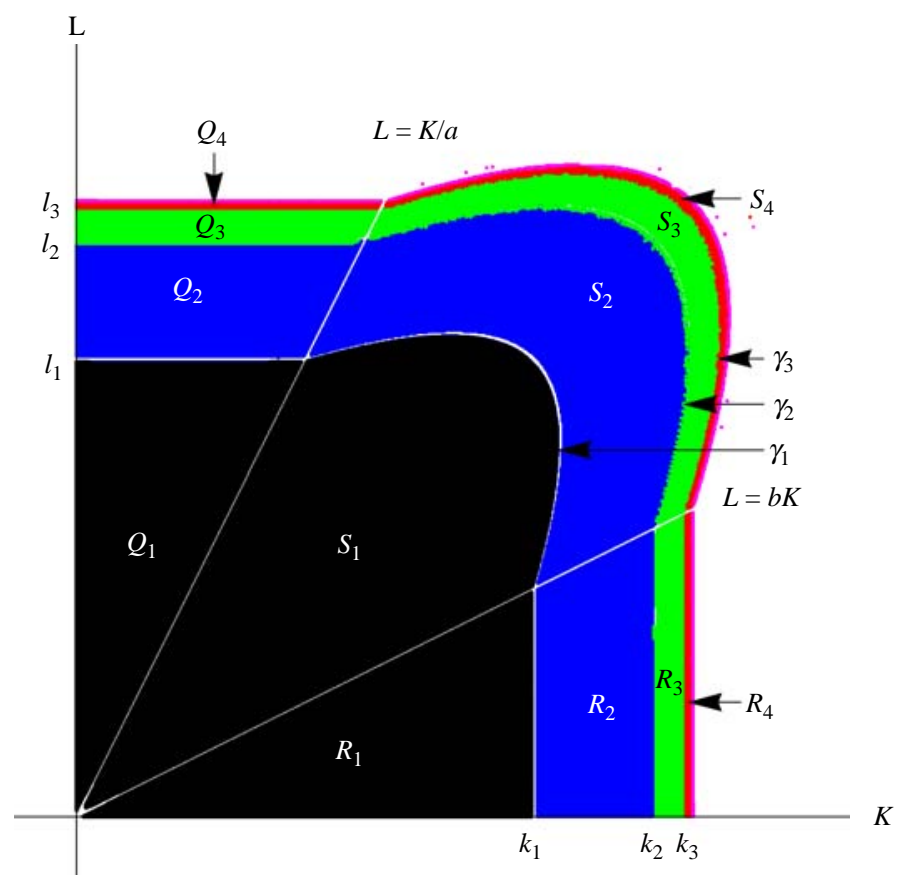

Figure 1. The stability regions and the bifurcation scenario of the Ricker competition equation in the parameter space $K-L$.

Open problem 1.3. Determine the basin of attraction of the periodic cycles of period $2^{n-1}$, $n=2,3, \ldots$ in regions $S_{n}$.

For the exclusion fixed points $(K, 0)$ and $(0, L)$, we have the following result from [2].

THEOREM 1.4. [2] For the Ricker competition equation (1), the following statements hold true:

(1) $(K, 0)$ is asymptotically stable if $0<K \leq 2$ and $L<b K$,

(2) $(0, L)$ is asymptotically stable if $0<L \leq 2$ and $L>K / a$.

Open problem 1.5.

(1) Determine the stability of the fixed point $(K, 0)$ when $K=2$ and $L=b K$. In this case, the eigenvalues of the Jacobian of the map of equation (1) are $\lambda_{1}=-1$ and $\lambda_{2}=1$.

(2) Determine the stability of the fixed point $(0, L)$ when $L=2$ and $L=K / a$. In this case, the eigenvalues of the Jacobian of the map of equation (1) are $\lambda_{1}=1$ and $\lambda_{2}=-1$.

\section{Logistic competition model}

In [1], the authors considered the following logistic competition model:

$$
\left\{\begin{array}{l}
x_{n+1}=\frac{a x_{n}\left(1-x_{n}\right)}{1+c y_{n}} \\
y_{n+1}=\frac{b y_{n}\left(1-y_{n}\right)}{1+d x_{n}}
\end{array},\right.
$$


where $x, y \in[0,1], c, d \in(0,1)$ and $a, b \in(0,4]$. The parameters $a$ and $b$ are called the intrinsic growth rates of species $x$ and $y$, respectively, and $c, d$ denote the competition of the species.

System (3) has the fixed points $(0,0),((a-1) / a, 0),(0,(b-1) / b)$ and $\left(x^{*}, y^{*}\right)$, where

$$
x^{*}=\frac{b(a-1)-c(b-1)}{a b-c d}, y^{*}=\frac{a(b-1)-d(a-1)}{a b-c d} .
$$

The Jacobian of system (3) at the fixed point $\left(x^{*}, y^{*}\right)$ is given by

$$
J=\left[\begin{array}{cc}
\frac{1-2 x^{*}}{1-x^{*}} & -\frac{c x^{*}}{a\left(1-x^{*}\right)} \\
-\frac{\mathrm{d} y^{*}}{b\left(1-y^{*}\right)} & \frac{1-2 y^{*}}{1-y^{*}}
\end{array}\right] .
$$

We make the assumption that

$$
b>1+\frac{d(a-1)}{a} \text { and } a>1+\frac{c(b-1)}{b},
$$

which insures that the fixed point $\left(x^{*}, y^{*}\right)$ lies in the positive first quadrant.

THEOREM 2.1. [1] The positive fixed point $\left(x^{*}, y^{*}\right)$ of the Logistic competition equation (3) is asymptotically stable if the following conditions hold:

$$
\begin{aligned}
& \frac{-c(b-c+b c) d^{2}+a^{3} b^{2}(2-b+2 d)+a(b-c+b c) d(3 b+c+c d)}{a b(-(1+c) d+a(1+d))(-b(1+c)+c(1+d))} \\
& +\frac{a^{2} b\left(2 b^{2}(1+c)+3 c(1+d)-b(3+5 d+c(5+4 d))\right)}{a b(-(1+c) d+a(1+d))(-b(1+c)+c(1+d))}<0
\end{aligned}
$$

and

$$
\begin{aligned}
& \frac{-c(b-c+b c) d^{2}+a^{3} b^{2}(3-b+3 d)-a d\left(-b^{2}\left(9+14 c+5 c^{2}\right)+c^{2}(1+d)\right)}{a b(-(1+c) d+a(1+d))(-b(1+c)+c(1+d))} \\
& -\frac{a d b c(8+4 c+4 d+3 c d)}{a b(-(1+c) d+a(1+d))(-b(1+c)+c(1+d))} \\
& +\frac{a^{2} b\left(3 b^{2}(1+c)+c\left(9+14 d+5 d^{2}\right)-3 b(3+4 d+4 c(1+d))\right)}{a b(-(1+c) d+a(1+d))(-b(1+c)+c(1+d))}>0 .
\end{aligned}
$$

Note that inequality (21) in [1], i.e.

$$
\frac{(b(-1+a-c)+c)(a(-1+b-d)+d)(a b-c d)}{a b(-(1+c) d+a(1+d))(-b(1+c)+c(1+d))}<0
$$

holds true under condition (4). This observation was not noted in [1].

Equivalently, the positive fixed point $\left(x^{*}, y^{*}\right)$ of equation (3) is asymptotically stable if $(a, b) \in \operatorname{Int}\left(S_{1}\right)$, where $S_{1}$ is the region depicted in Figure 2. Note that the curves $\tau_{1}$ and $\tau_{2}$ 
in Figure 2 are defined as

$$
\tau_{1}=\left\{(a, b) \in \mathbb{R}_{+}^{2}: b=1+\frac{d(a-1)}{a}\right\} \text { and } \tau_{2}=\left\{(a, b) \in \mathbb{R}_{+}^{2}: a=1+\frac{c(b-1)}{b}\right\}
$$

ConjeCture 2.2. The positive fixed point $\left(x^{*}, y^{*}\right)$ of the logistic competition model (3) is globally asymptotically stable if $(a, b) \in \operatorname{Int}\left(S_{1}\right)$.

Open problem 2.3. Determine the basin of attraction of the periodic cycles of period $2^{n-1}$, $n=2,3, \ldots$ in regions $S_{n}$ in Figure 2 .

For the fixed points $((a-1) / a, 0)$ and $(0,(b-1) / b)$, we have the following result:

THEOREM 2.4. [1] The following statements hold true:

(1) The fixed point $((a-1) / a, 0)$ of equation (3) is asymptotically stable if $1<a \leq 3$ and $1<b<1+(d(a-1) / a)$ and is unstable if $1<a<3$ and $b=1+(d(a-1) / a)$,

(2) The fixed point $(0,(b-1) / b)$ of equation (3) is asymptotically stable if $1<b \leq 3$ and $1<a<1+(c(b-1) / b)$ and is unstable if $1<b<3$ and $a=1+(c(b-1) / b)$.

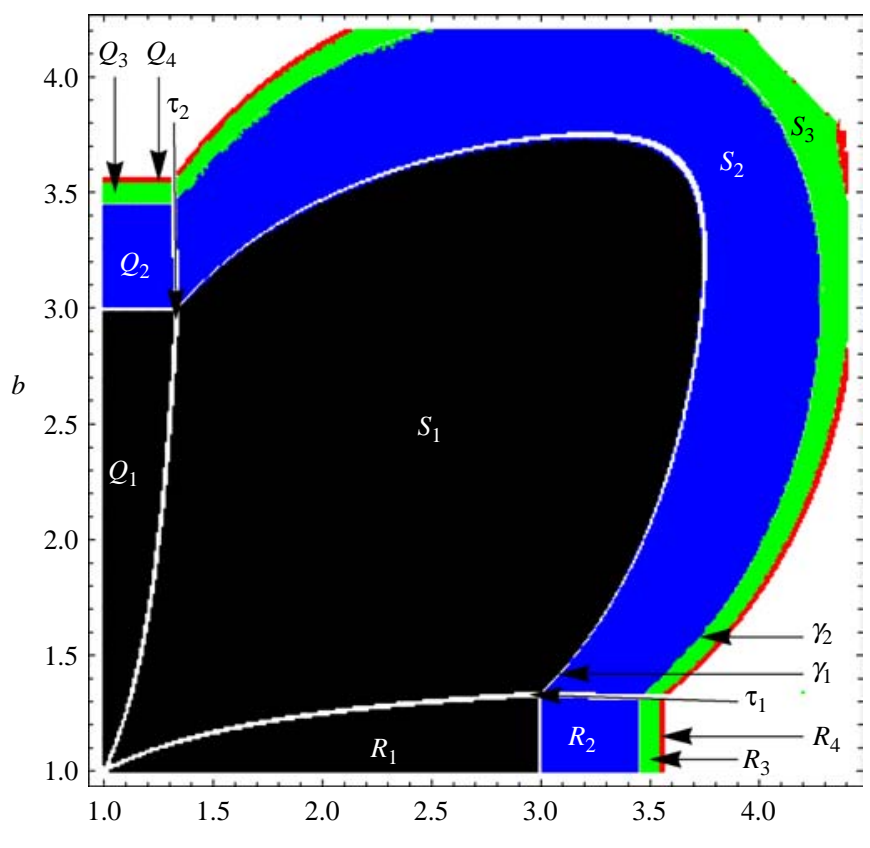

Figure 2. The stability regions and the bifurcation scenario of the competition logistic model in the parameter space $a-b$. 
Open problem 2.5 .

(1) Determine the stability of the fixed point $((a-1) / a, 0)$ of equation (3) if $a=3$ and $b=1+(d(a-1) / a)$.

(2) Determine the stability of the fixed point $(0,(b-1) / b)$ of equation (3) if $b=3$ and $a=1+(c(b-1) / b)$.

\section{Note}

1. Email: saber.elaydi@trinity.edu

\section{References}

[1] M. Guzowska, R. Luís, and S. Elaydi, Bifurcation and invariant manifolds of the logistic competition model, J. Difference Equ. Appl., accepted.

[2] R. Luís, S. Elaydi, and H. Oliveira, Stability of a Ricker-type competition model and the competition exclusion principle, Submitted. 